\title{
Some psychosocial therapies may reduce depression, aggression, or apathy in people with dementia
}

Verkaik R, van Weert JCM, Francke AL. The effects of psychosocial methods on depressed, aggressive and apathetic behaviours of people with dementia: a systematic review. Int J Geriatr Psychiatry 2005;20:301-14.

Does psychosocial therapy reduce depression, aggression, and apathy in people with dementia?

\section{METHODS}

$-4$

Design: Systematic review.

Data sources: PubMed, Cochrane CENTRAL/CCTR, Cochrane Database of Systematic Reviews, PsyclNFO, EMBASE, CINAHL, INVERT, NIVEL, Cochrane Specialised Register CDCIG, SIGLE, Cochrane Database of Abstracts of Reviews of Effectiveness (September 2002 to February 2003); hand searches of review articles and reference lists.

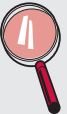

Study selection and analysis: Eligible studies were randomised controlled trials (RCTs), non-RCTs, and crossover trials comparing psychosocial versus control interventions in people with dementia diagnosed according to DSM-III, DSM-IV, or ICD-10 criteria. The psychosocial interventions were: behaviour, psychomotor, and art therapy, supportive psychotherapy, validation/integrated emotion oriented care, multisensory stimulation/snoezelen, simulated presence therapy, reminiscence, gentle care, passivities of daily living (PDL), reality orientation, skills training, and activity or recreational therapy. Data were extracted on age, sex, type of dementia, diagnostic criteria, severity and duration of dementia, type of psychosocial and control intervention, outcome measures, and study duration. Data were not pooled due to the heterogeneity of study interventions and outcome measures.

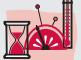

Outcomes: Depression (Hamilton Depression Scale, Cornell Scale for Depression in Dementia, and Beck Depression Inventory); aggression (Dutch Assessment Scale for Elderly Patients-aggression subscale); apathy (Dutch Behaviour Observation Scale for psychogeriatric Inpatients - apathetic behaviour subscale).

\section{MAIN RESULTS}

Nineteen studies met inclusion criteria. Depression: one high quality RCT ( 72 people) found that behaviour therapy (pleasant events and problem solving dimensions), administered by a trained caregiver, reduced depression in people with Alzheimer's disease and a major or minor depressive disorder (DSM-III-R criteria), compared with an untrained caregiver or a caregiver given standard information by a therapist (no further data reported). Aggression: one high quality RCT (43 people) found that psychomotor therapy significantly reduced aggression compared with activity therapy in people with Alzheimer's disease, who were "functionally disordered", but mobile, and living in a residential nursing home (no further data reported). Apathy: one high quality RCT (66 people) found that multisensory stimulation/snoezelen therapy significantly reduced apathy compared with activity therapy or no therapy, in people with moderate to severe dementia (MMSE 0-17; no further data reported). The review found no evidence of the effect of behaviour therapy (pleasant events and problem solving dimensions), psychomotor therapy, or multisensory stimulation, on the other outcomes of interest. The review also found limited or no evidence of the effect of other psychosocial interventions on depression, aggression, or apathy. For correspondence: R VerkaikMSc, NIVEL, PO Box 1568, 3500 BN Utrecht, the Netherlands; r.verkaik@nivel.nl

Sources of funding: The Netherlands Organisation for Health Research and Development (ZonMW).

\section{CONCLUSIONS}

Behaviour therapy administered by a trained caregiver reduced depression in people with Alzheimer's disease and a major or minor depressive disorder. Psychomotor therapy reduced aggression compared with activity therapy in people with Alzheimer's disease. Multisensory stimulation/snoezelen therapy reduced apathy compared with activity therapy or no therapy in people with moderate to severe dementia. However, there is limited or no evidence of the efficacy of other psychosocial interventions on depression, aggression, or apathy.

Commentary

7 he neuropsychiatric symptoms of dementia, also known as behavioural and psychological symptoms of dementia (BPSD) are common, serious problems that effect the quality of life for both the patient and their caregivers. These include symptoms such as depression, apathy, agitation, aggression, hallucinations, and delusions. In the absence of therapies that can arrest or reverse the underlying neurodegenerative process, clinicians treating people with dementia are often required to provide specific interventions for these problems.

Treatment has broadly been conceptualised as pharmacological and non-pharmacological in approach, with the latter including environmental, psychological, and social forms of therapy. Clinical practice guidelines frequently suggest the use of non-pharmacological approaches before resorting to the use of psychotropic agents such as antipsychotics, ${ }^{1}$ recommendations that will likely be strengthened given recent concerns about the safety of these medications. ${ }^{2}$ But what is the evidence that non-pharmacological approaches work for BPSD?

Using the Cochrane Collaboration methodology, Verkaik et al systematically reviewed some of these approaches. They chose to examine studies that could be categorised along the 10 psychosocial approaches described by the American Psychiatric Association's clinical practice guideline for dementia. ${ }^{3}$ These were then divided into four methods: behaviour oriented (for example, behavioural therapy), emotion oriented (for example, supportive psychotherapy, multisensory stimulation, reminiscence), cognition oriented (for example, reality orientation), and stimulation oriented (for example, activity therapy, art therapy). Although this approach was not unreasonable, it did mean that potentially useful non-pharmacological approaches like music therapy, aromatherapy, and bright light were not included. Outcome measures focused on depression, aggression, or apathy. A best evidence approach would have ideally focused on randomised controlled trials (RCTs), but the authors had to include non-randomised controlled trials given the paucity of studies. Even so, there were only 19 studies that could be included, 10 of which were RCTs, and only two with sample sizes greater than 100. Despite the authors' overly optimistic conclusions, none of the interventions were supported by a minimum of two positive RCTs for the same target symptom. Although clinicians should still consider the use of non-pharmacological approaches for the treatment of BPSD, we urgently need high quality studies to delineate which of these varied approaches works best for which patients and which target behaviours.

Nathan Herrmann MD FRCPC Professor, and Head of the Division of Geriatric Psychiatry, University of Toronto, Toronto, Ontario, Canada

1 Herrmann N. Recommendations for the management of behavioral and psychological symptoms of dementia. Can J Neurol Sci 2001;28(Suppl 1):S96-S107

2 Herrmann N, Lanctot KL. Do atypical antipsychotics cause stroke? CNS Drugs 2005; 19:91-103

3 American Psychiatric Association. Practice guideline for the treatment of patients with Alzheimer's disease and other dementias of late life. Am J Psychiatry 1997; 154:1-35. 